\title{
CRM e a web 2.0 no setor da saúde: um estudo de caso em uma clínica de medicina dentária de Lisboa (Portugal)
}

Este estudo teve como objetivo apresentar os benefícios do Custumer Relationship Management (CRM) e da Web 2.0 na relação de uma Clínica de Medicina Dentária com seus clientes e na gestão do negócio. Desta forma, foi realizado uma pesquisa qualitativa através de estudo de caso em uma Clínica de Medicina Dentária de Lisboa(Portugal), que logo no primeiro ano de implementação obteve resultados significativos com a utilização desta metodologia de gestão. Os resultados do estudo demostram que a adoção do CRM e a utilização da Web 2.0 contribuíram para: o Aumento do faturamento; Conhecer o perfil do cliente, Aumento da carteira de clientes; Facilitar o trabalho dos profissionais e, Ter acesso às informações em qualquer lugar. Com o resultado do estudo é possível concluir que o diferencial competitivo no setor da saúde pode estar na gestão do relacionamento com o cliente e no uso de novas tecnologias que dão suporte a essa gestão do negócio. Foi possível também com o estudo criar um perfil dos clientes da clínica que são: homens e mulheres, entre 20 anos e 40 anos da classe B, C que se preocupa com a saúde dentária e sua aparência.

Palavras-chave: Marketing de relacionamento; CRM; Setor da Saúde e Web 2.0.

\section{CRM y web 2.0 en el sector de la salud: un estudio de caso en una clínica dental de Lisboa (Portugal)}

Este estudio tuvo como objetivo presentar los beneficios de Custumer Relationship Management (CRM) y de la Web 2.0 en la relación de una Clínica de Odontología con sus clientes y en la gestión del negocio. Por lo tanto, se realizó una investigación cualitativa a través de estudio de caso en una clínica de odontología en Lisboa (Portugal), que en el primer año de aplicación ha logrado resultados significativos con el uso de esta metodología de gestión. Los resultados del estudio demuestran que la adopción del CRM y la utilización de la Web 2.0 contribuyeron a: el aumento de la facturación; Conocer el perfil del cliente, Aumento de la cartera de clientes; Facilitar el trabajo de los profesionales y, Tener acceso a la información en cualquier lugar. Con el resultado del estudio es posible concluir que el diferencial competitivo en el sector de la salud puede estar en la gestión de la relación con el cliente y en el uso de nuevas tecnologías que dan soporte a esa gestión de negocio. En el estudio se creó un perfil de los clientes de la clínica que son: hombres y mujeres, entre 20 años y 40 años de la clase B, C, que se preocupa por la salud dental y su apariencia.

Keywords: Marketing de relaciones; CRM; Sector de la Salud y Web 2.0

Topic: Marketing e Estratégias Mercadológicas

Reviewed anonymously in the process of blind peer.
Received: 11/01/2017

Approved: 12/03/2017
Mônica Maria Liberato

Faculdade Maurício de Nassau, Brasil

http://lattes.cnpq.br/6716169981657880

focoeadcursos@gmail.com
Referencing this:

LIBERATO, M. M.. CRM e a web 2.0 no setor da saúde: um estudo de caso em uma clínica de medicina dentária de Lisboa (Portugal). Revista Brasileira de Administração Científica, v.8, n.1, p.72-81, 2017. DOI: http://doi.org/10.6008/SPC2179-684X.2017.001.0006 


\section{INTRODUÇÃO}

Nos últimos anos, com a crescente concorrência, muitas organizações vêm identificando a necessidade de estarem mais próximas dos seus consumidores, tornando suas estratégias um diferencial competitivo nesse mercado tão acirrado. E como consequência o Customer Relationship Management (CRM) passou a ser uma das estratégias organizacionais mais utilizada para atingir esse objetivo (BULL, 2003; LIBERATO, 2014).

No caso de organizações com pontos de contato online com os clientes, a integração das ferramentas de CRM dentro da estrutura operacional oferece a possibilidade de identificar, avaliar e definir claramente os segmentos dos principais clientes em termos de rentabilidade, e construir uma ponte na captação de novos clientes. Já a integração dos Customers Databases com as ferramentas Data Mining e software da gestão do cliente permitem gerir de forma eficaz a interação com o cliente, a nível individual (AURILI et al., 2013).

Essa interação (cliente - empresa) é registada no histórico de transações, para criar uma base melhorando a segmentação dos clientes em campanhas de marketing no futuro. Como resultado da adoção do CRM, a relação cliente - empresa será mais eficaz, com uma maior satisfação do cliente, maior lealdade e maior lucratividade para a empresa (GURAU et al., 2003).

É pela necessidade de identificar e aplicar as muitas possibilidades de uso do CRM e da Web 2.0 nas relações com os clientes e a eficácia do uso dessa plataforma para o setor da saúde, demonstram a importância de trabalhos que possam discutir a relevância do tema, diante da impressionante marca alcançada pelo volume de informações produzido no planeta em 2013 (AURILI et al., 2013) em meios digitais - de 1,8 zettabyte (1 zettabyte é igual a 1.000.000.000.000.000.000.000 bytes). Discussão está que possibilita ter uma visão mais ampla do paciente como um cliente que pode ser fidelizado. Pensando nisso, o objetivo deste estudo é apresentar os benefícios da utilização CRM e da Web 2.0 na relação de uma Clínica de Medicina Dentária com seus clientes e na gestão do negócio.

\section{REVISÃO TEÓRICA}

\section{Customer Relationship Management - CRM}

O termo CRM tem vários significados, segundo alguns autores, mas a sua essência está sempre relacionada com a prática empresarial com foco nos clientes. Já para algumas pessoas nas organizações o CRM é a mesma coisa que o Database Marketing, outras acreditam que o CRM não passa de um processo de Marketing ou um acontecimento de Tecnologias de Informação (TI), ou mesmo um programa de fidelidade. Segundo Buttle (2008), essa falta de conhecimento é um dos fatores críticos que dificultam o sucesso na adoção do CRM.

Para Urbanowicz (2008) o CRM pode ser visto como uma estratégia que respeita os aspectos internos e externos das organizações com o objetivo de criar valor para a empresa e para os clientes. É uma estratégia de negócios para maximizar o valor para os acionistas por meio de atrair e manter os clientes. Nesse contexto os elementos principais são o foco nos clientes mais importantes e a manutenção de um relacionamento de 
longo prazo. E para melhorar esse valor é preciso construir e fortalecer o relacionamento com o cliente, e assim é possível maximizar a produtividade da empresa e também o valor dos acionistas (PAYNE, 2006). Buttle (2008) apresentou em seu trabalho uma definição que se assemelha a de Urbanowicz (2008) quando ele diz que o CRM é uma estratégia de negócios: “É a estratégia central de negócios que integra processos e funções internas e redes externas, para criar e entregar valor aos clientes rentáveis. Se baseando em dados de clientes de alta qualidade e habilitado pela Tecnologia da Informação" (BUTTLE, 2008).

Como diz Buttle (2008) e Urbanowicz (2008), o CRM está relacionado especificamente na estratégia central dos negócios da empresa, na criação do valor de clientes, no uso inteligente dos dados e tecnologias, na aquisição e conhecimento dos clientes e na disseminação desse conhecimento às partes interessadas, para assim desenvolver um relacionamento de longo prazo com os clientes atuais. Pois segundo os autores manter os clientes pode ser mais rentável do que prospectar novos clientes. Nesse contexto, o objetivo central do CRM é maximizar o valor da vida de um cliente para a organização.

Desta maneira é possível aumentar a retenção do cliente com o Marketing de Relacionamento, assim é mais fácil construir um relacionamento cooperativo e duradouro, já que as preferências dos clientes podem ser identificadas. E quanto mais os clientes são mantidos, maior é a oportunidade de uma venda cruzada (PEPPARD, 2000).

Para outros autores, como Liberato (2014), Glick (2007) e Tehrani (2000), o CRM vai além do uso da tecnologia de armazenamento de dados, é uma filosofia de relacionamento entre os clientes e empresa, onde os clientes rentáveis tem mais atenção e privilégios. Não se eximindo de fortalecer as relações com os menos rentáveis, muito menos sem deixar de lado os novos clientes, através da qualificação e análise dos dados destes clientes. Portanto, o CRM tem o foco mais filosófico e estratégico do que tecnológico, isso significa que é importante manter o relacionamento entre os clientes e a empresa. Já quando o CRM tem enfoque na tecnologia visa apenas a integração das Sales Force Administration, Data Mining, Data Warehouse, Call Center, Contact Center, sem se preocupar com a satisfação dos clientes.

\section{Customer Relationship Management na Saúde}

Quando se fala em informatização de sistemas do Serviço Médico Hospitalar (SIH), observa-se a preocupação do planejamento estratégico que decidirá o enfoque da organização de saúde, com o mesmo propósito das instituições de serviço dos demais segmentos - o consumidor - para tanto se faz necessário obter o maior número de dados e transformá-los em informações mercadológicas (GALLO et al., 2006). Portanto, a informatização de sistemas na saúde deve ser entendida como apoio na tomada de decisão da gestão, na organização e na avaliação dos vários níveis que constituem os Sistemas de Saúde (KRAUS et al., 2006).

De acordo com Gallo et al. (2006), as vantagens da implantação do CRM no setor da saúde são: a possibilidade do conhecimento do perfil do cliente (a delimitação das áreas geográficas de maior procura para Instituição) e a determinação do perfil dos serviços de maior oferta pela Instituição. Já para Kraus et al. 
(2006), o “CRM além de identificar o perfil do cliente também possibilita identificar como se dá a comunicação entre os clientes, a instituição e a personalização do atendimento".

As principais e primeiras aplicações do Sistema de Informação (SI) no setor da saúde deram-se em caráter administrativo, financeiro ou contabilístico (MARTINEZ et al., 1982). Antes da aplicação do Sistema de Informação Hospitalar (SIH) os processos eram compostos por vários documentos de papel sem maior compromisso. As únicas preocupações estavam em reunir informações para emissão de relatórios nas análises estatísticas e em outros documentos em papel que agrupados formavam o prontuário do paciente que eram simplesmente usados, armazenados e consultados.

Os dados clínicos que deveriam ser o principal motivo da existência do SIH, às vezes eram esquecidos ou mal utilizados (LAUDON et al., 1999). A ideia principal do SIH é "promover a automação dos processos, para buscar eficiência e produtividade" (LEITE et al. 2009). É possível entender como funciona o processo de um sistema SIH na figura 1, onde Gallo et al. (2006) dão como exemplo de fluxograma no seu trabalho, o Diagrama da interatividade de processo criado pelo autor McKenna (1996).

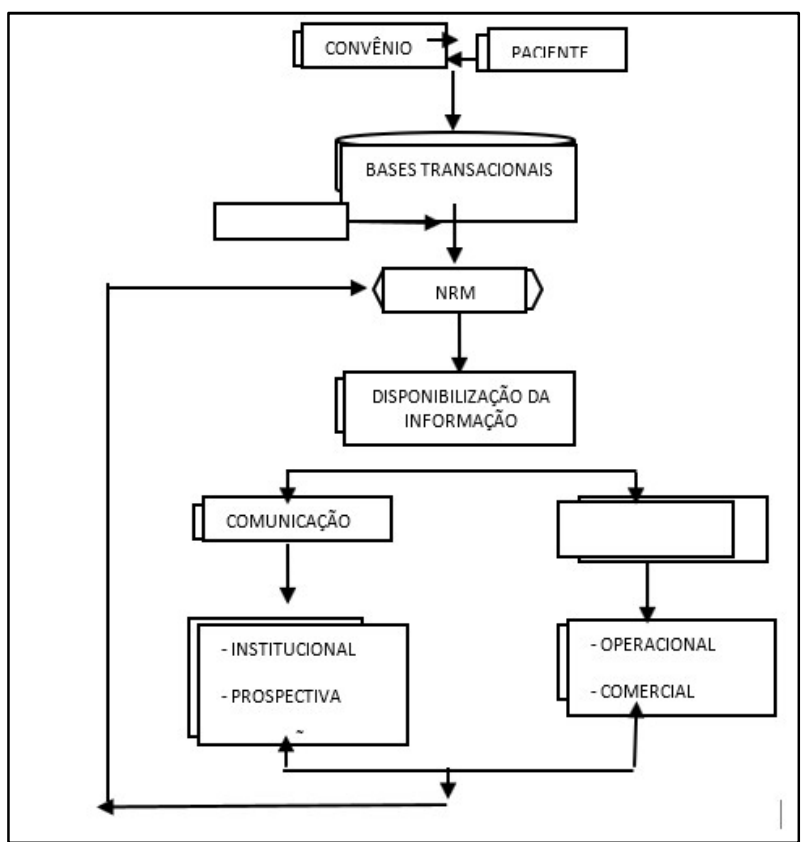

Figura 1: Diagrama da interatividade de processo. Fonte: McKenna (1996, citado por GALLO et al., 2006)

Segundo Leite et al. (2009), a automação hospitalar de uma forma geral poder ser observada sob duas perspectivas: A rede de informação que é composta pelos SIH (prontuário electrónico, marcação de consultas, sistema de internamento, sistema de laboratório, outros); e outra perspectiva é a rede de controle que é composto pelos sistemas utilizados no acompanhamento de pacientes.

A tendência do setor da saúde é utilizar a tecnologia Ethernet em seu Sistema de Informação (SHIN et al., 2000). Essas características facilitam a integração com uma comunicação transparente entre os pontos de contatos da rede. E tudo isso só é alcançado porque a tecnologia Ethernet tem baixo custo e fácil penetração no mercado (BRITO et al., 2004).

Gallo et al. (2006) concluíram que a partir da interação entre as instituições de saúde e seus clientes podem-se propor programas de fidelização através de ações de vendas cruzadas, que passaram a incorporar 
no orçamento anual os investimentos em marketing e comunicação para a divulgação dos serviços oferecidos, bem como, na criação de novos canais a fim de fortalecer o relacionamento com os clientes. Salientou também que com a implantação do CRM possibilitou a abertura de novos nichos de mercado para o hospital.

\section{Redes Sociais}

As redes sociais é um fenômeno (COOKE et al., 2008), e devido a este fenômeno está surgindo uma população que cada vez mais disposta a compartilhar as suas experiências de vida com seus amigos virtuais para serem avaliados, e permitir que a sua reputação seja construída através destas avaliações (COOKE et al., 2008). E as organizações com interesses nessa população cibernética têm crescido a cada dia.

Os especialistas de marketing estão aprendendo que as comunidades online podem promover mensagens positivas sobre os produtos/serviços, fortalecer a marca, e recrutar mais membros, pode proporcionar grandes vantagens competitivas (MORAN et al., 2010).

No mundo virtual, as pessoas podem formar comunidades online através de modos de combinações que podem ser, um-para-um (por exemplo, e-mail e mensagens instantâneas), um-para-muitos (páginas Web, Facebook e Blogs) e muitos-para-muitos (Wikis) (COOKE et al., 2008). Os objetivos principais dessas comunidades online incluem o conhecimento de mercado, geração de ideias, a lealdade com o cliente, ampliação da palavra boca a boca, e suporte ao cliente (MORAN et al., 2010). O desenvolvimento e manutenção das comunidades online são desafiadores para as organizações, que cometem constantemente erros. Os erros mais comuns são apresentados por Moran et al. (2010) em seu trabalho, são eles:

'Construa e eles virão': Alguns patrocinadores de comunidade pensam que pela simples criação de uma comunidade online garante que as pessoas vão aderi-la;

A Síndrome 'não inventado aqui': Alguns patrocinadores de comunidade ignoram a presença de outras comunidades, em vez disso, tente criar a sua comunidade 'própria';

'Vamos mantê-lo pequeno para que não se mover nem um bocadinho': Muitos dos esforços que a comunidade forma são demasiados pequenos para fazer uma diferença sensível ao processo do negócio que estava destinado a melhorar.

'A minha empresa é mais inteligente do que eu': Algumas empresas patrocinadoras acreditam que as tribos querem falar com a empresa. Comunidades bem sucedidas indicam, no entanto, que as pessoas gostam de falar com as pessoas identificáveis e estabelecer relações mais fortes com os seres humanos do que com os pontos de vista organizacional.

\section{A Web 2.0 e o Setor da Saúde}

A Web 2.0 é composto das plataformas de software social, que incluem as mídias sociais como: Wikis, Blogs, Podcasts e Vodcasts, Twitter, Myspace, Youtube, Facebook, dentre outras. Essas plataformas estão crescendo de forma assustadora proporcionando as empresas de diversos segmentos, formas inovadoras de se relacionar com seus clientes. Conceitualmente, a Web 2.0 surgiu no vocabulário em 2004, e o termo Web 2.0 é um reflexo do movimento de conteúdo estático, centrado no fornecedor e aplicações para a abordagem mais interativa (ALKHATEEB et al., 2008; COOKE et al., 2008). 
As oportunidades com a Web 2.0 têm sido exploradas por empresas farmacêuticas e instituições médicas. Recentemente, o termo 'paciente 2.0' foi introduzido para descrever Web 2.0 utilizada pelos pacientes. Um exemplo de um site de relacionamento do paciente 2.0 inclui grupos temáticos de apoio, como a cura da diabetes no grupo do MySpace. O site pode ser focado em uma doença específica/tratamento ou mesmo em assistir o paciente/cliente. Se for bem aproveitados os Blogs, Podcasts, Redes Sociais e Wikis podem ser usados pelas empresas no setor da saúde para a interação ativa entre os médicos e os pacientes (ALKHATEEB et al., 2008).

\section{METODOLOGIA}

A metodologia utilizada para o estudo foi a qualitativa, através de um estudo de caso realizado em uma Clínica de Medicina Dentária na cidade de Lisboa (Portugal). Segundo Neves (1996), o estudo de caso tem a possibilidade de analisar detalhes sobre o ambiente, setor ou qualquer situação pesquisada, facilitando assim, a construção da realidade da instituição objeto de estudo.

Para a coleta de dados foi utilizado entrevistas semiestruturadas com o Presidente da empresa, dois médicos e o Consultor de CRM que implantou a metodologia na clínica. As entrevistas pessoais foram gravadas e posteriormente transcritas para análise de conteúdo. Além disso, foram feitas entrevistas com 47 clientes através de um questionário construído no programa Google docs e o link do questionário foi enviado para os e-mails dos mesmos e depois de recebidos foi feita uma análise dos resultados, além da análise de documentos disponibilizados pela empresa.

\section{Descrição da empresa objeto de estudo}

A Clínica de Medicina Dentária, objeto deste estudo, aqui identificada como Clínica Alfa, é uma empresa do setor da saúde que presta serviços na área de medicina dentária, está localizada em Lisboa, Portugal e tem um volume médio de negócios de 270 mil euros. Fundada em 2008 por dois brasileiros, a Clínica Alfa conta com uma equipe de sete médicos com experiência de mais de 10 anos e quatro colaboradores administrativos.

A prioridade da Clínica é a satisfação dos clientes através do investimento em tecnologia de ponta. A sua infraestrutura é de alto nível, com cadeiras de cinema e projetor HD 3D com tela de mais de 140 polegadas que transmite filmes para entreter os clientes. Tem também uma brinquedoteca na recepção para as crianças. Com todo o diferencial proposto pela Clínica Alfa, criou-se em Portugal um novo conceito de relacionamento de clientes na área de medicina dentária.

\section{RESULTADOS E DISCUSSÃO}

\section{O Sistema de Customer Relationship Management}

O sistema de CRM utilizado pela clínica 'TWDent (portal) + Odontouch' foi desenvolvido pela empresa Towntech Corporation, é um sistema de CRM online que consiste em um conjunto de módulos que funciona de forma online, conforme se visualiza na figura 2, onde recolhe os registos dos pacientes da Web 2.0 (portal 
e redes sociais) e toda informação é registada pelos colaboradores da clínica em uma base de dados, e as informações são recebidas em tempo real por todas as pessoas que tem acesso ao sistema com toda segurança, pois todos os dados têm criptografia SSL (Secure Sockets Layers) e não é necessária qualquer instalação física nos computadores da clínica.

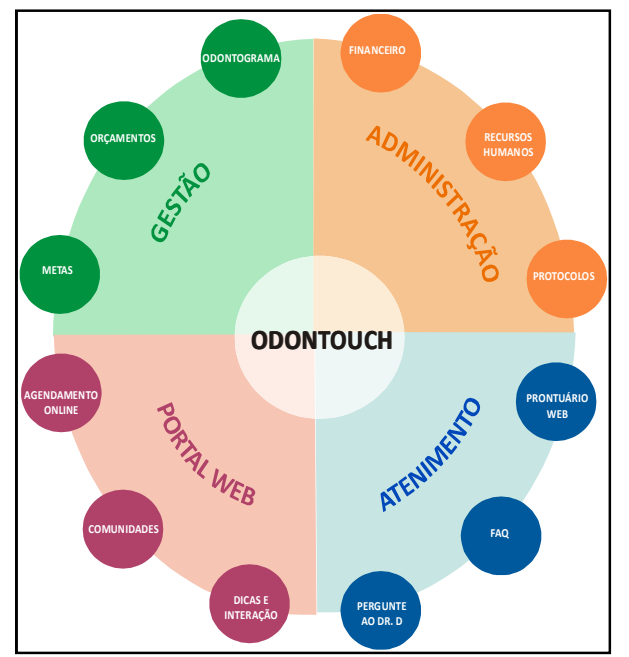

Figura 2: Arquitetura do Sistema 'Odontouch'.

O sistema de CRM online, além do registo dos dados do paciente possibilita acompanhar a evolução do tratamento, bem como o acesso das radiografias do paciente pelo médico. Possibilita ainda, identificar a quantidade de acesso que as redes sociais da clínica tiveram. Os dados dos clientes que são alimentados no sistema, são recolhidos antes em uma ficha de papel preenchida pelo próprio cliente, e só depois que a recepcionista passa as informações para o sistema. A Clínica Alfa começou a trabalhar com o CRM online em 2012 e antes era tudo feito manualmente, mesmo antes da adoção do sistema, a clínica já tinha adotado a filosofia do CRM.

Antes da aquisição do sistema de CRM online, o objetivo da clínica era atender o público apenas da classe " $\mathrm{A}$ ", visto que em Portugal já tinha muitas clínicas populares, mas, depois da implantação do sistema e adesão da clínica nas redes sociais, a procura passou a ser de pessoas de todas as classes, até as celebridades como: jogadores de futebol, modelos e artistas. 'O mais importante é que não existe distinção no tratamento, pois cada cliente recebe um cartão de fidelização', diz o presidente da clínica.

No início a clínica Alfa estava presente em oito comunidades (Facebook, Orkut, Myspace, Twitter, Blog, Hi5, Youtobe e Netlog). Mas segundo o Presidente, as comunidades que tinha mais acesso na época eram: Facebook e o Orkut e a menos utilizada é a Netlog. 'Hoje reduzimos para quatro apenas, Facebook, Youtobe, Twitter e Myspace'. As redes socias bem utilizadas pelas empresas no setor da saúde podem fortalecer o relacionamento com os clientes, promover os produtos/serviços e atrair novos clientes (ALKHATEEB et al., 2008).

A Clínica Alfa utiliza as parcerias feitas com empresas como a Fundação Salvador Caetano, Holmes Places, Colgate e outros, e a newsletter que manda para os parceiros e os clientes, para além de divulgar os serviços e promoções da clínica e atrair seguidores para as redes sociais. Outra forma de atrair seguidores para as redes sociais é através de um kit que é entregue aos clientes e parceiros com material da clínica e um 
pendrive com vídeos institucionais. Outro trabalho muito importante feito pela clínica são as ações educativas sobre a saúde dentária nas escolas em parceria com a Colgate.

\section{Aprendizados obtidos com o CRM online}

Desde que a Clínica Alfa utiliza a Web 2.0 como estratégia de CRM, houve muito aprendizado, principalmente com a gestão do negócio, além de fechar boas parcerias também, como por exemplo, a parceria com a Turma do Bem, a maior organização Brasileira que trata de crianças carentes até os 18 anos na área odontológica e que está começando a se fortalecer em Portugal. No Brasil, a Turma do Bem faz parcerias com as clínicas de saúde dentária para que forneçam tratamentos sem custos para estas crianças em troca de publicidade. A Turma do Bem conheceu a clínica através do Facebook. Hoje, a clínica faz tratamentos em 20 crianças por mês inteiramente grátis.

A Clínica Alfa com o objetivo de fortalecer o relacionamento com os clientes utiliza os sites de compras coletivas Groupon e Letsbonus, chegando a cento e trinta pacotes de tratamentos e limpezas dentárias a preços promocionais. "Os benefícios dessas promoções é fazer com que as pessoas conheçam as instalações e os equipamentos da clínica e depois tornarem-se clientes', afirmou o Presidente da Clínica.

\section{Benefícios obtidos com a implantação do CRM online}

Os benefícios de utilizar um sistema de CRM para a gestão do relacionamento dos clientes através da Web 2.0 são muitos, entre eles: ter acesso aos clientes em qualquer parte do mundo, principalmente no sistema de franchising, onde a clínica pode entrar em contato com as futuras clínicas de franchising em tempo real. A comunidade, segurança e a rapidez em que as informações são passadas e a inexistência de fichas em papel são outros benefícios importantes. Em termos de retorno financeiro a clínica Alfa teve um aumento de 60\% a 70\% no seu faturamento após a utilização do CRM e da Web 2.0 como estratégia de negócio.

Com as redes sociais e o portal as pessoas conhecem melhor os serviços da clínica, elas tem acesso a todas as informações dos tratamentos, os vídeos explicativos e o vídeo 360 graus da clínica para facilitar a localização do prédio. Os clientes têm acesso a uma área pessoal no site com seu tratamento e agenda da próxima consulta. $O$ único custo que a empresa teve com a adoção do CRM online foi a contratação de um consultor que criou o sistema, o layout do portal e também é o responsável por toda padronização do material que está sendo feito para a abertura do franchising. Os médicos têm muitos benefícios, desde do acesso às fichas de tratamento dos pacientes e a sua agenda, em qualquer lugar com acesso à internet. As únicas dificuldades estão na parte da informática e no período de adaptação ao sistema.

'São tantos benefícios, em qualquer canto do mundo sei a minha agenda, sei que paciente, sei que procedimento vou fazer, vejo as radiografias online, eu já me planejo para atender aquele paciente mesmo estando longe', afirmou a Dra. Maria José'. Na mesma linha de raciocínio, 'Com o acesso online da agenda podemos planear melhor o nosso dia - a - dia. E o uso da tecnologia que a clínica oferece é mais valia, porque podemos mostrar através da câmara o tratamento, que é transmitido em tempo real no ecrã ao paciente e 
a cadeira que dá massagens no paciente enquanto ele está a ser tratado ajuda a tirar o nervosismo', disse a Dra. Bárbara Martins'.

\section{Resultados dos Inquéritos com os Clientes}

Quanto ao gênero dos clientes entrevistados observa-se que a maioria é feminino totalizando $72 \%$ da amostra, enquanto apenas $21 \%$ são do sexo masculino, demonstrando o cuidado que as mulheres têm com a saúde dentária, bem como com a sua aparência. A maioria dos entrevistados estão na faixa etária entre 21 anos e 40 anos, porém existe um público acima de 40 anos considerável, que merece ser trabalhado.

No que se refere à escolaridade os inquiridos tem em sua maioria ensino secundário (34\%) e ensino superior (36\%), esse dado dá uma direção para uns dos focos que a clínica deve ter em suas ações de marketing. Observa-se também que tem uma pequena percentagem de clientes com mestrados e doutorados (13\%). Em relação de como os clientes conheceram a clínica Alfa, a maioria (49\%) afirma que foi através de pesquisa na internet e redes sociais.

Outras formas foram o Groupon, o Lestbonus e indicações de amigos e família. 70\% dos entrevistados afirmaram que gostam da forma que a Clínica Alfa se relaciona com seus clientes, como é possível identificar nos depoimentos de alguns clientes, como uma senhora que afirma: 'Gosto do profissionalismo com que sou tratada'; e um outro cliente que disse: 'Durante os últimos dois anos tenho feito tratamentos dentários na clínica e não tenho razão de queixas. Indico sempre a quem pedir informações'; além disso, um outro cliente afirmou: 'Eu gosto muita da Clínica Alfa'.

Apesar de a Clínica Alfa fazer parte de oito comunidades, $77 \%$ dos entrevistados responderam que apesar de conhecer as redes sociais da clínica Alfa, não seguem nenhuma, 15\% são seguidores do Facebook, 4\% do Orkut e 4\% do Myspace. Esse resultado só corrobora a ideia de que não é necessário estar presente em todas as redes sociais, apenas aquela ou aquelas que mais se enquadra com o perfil dos clientes da clínica. Pois segundo Moran et al. (2010), as redes sociais tem o poder de promover os produtos/serviços de forma bastante positiva. Em relação as informações contidas nas redes sociais da Clínica Alfa, $49 \%$ dos entrevistados concordaram que as informações são úteis, 23\% acham indiferente e 26\% acham que não são úteis.

\section{CONSIDERAÇÕES FINAIS}

A adoção do CRM online e a utilização da Web 2.0 como estratégia para aprimorar a relação com o cliente possibilitou a Clínica Alfa obter mais informações dos seus clientes, e a forma que essas informações foram utilizadas estimulou a fidelização dos mesmos aos serviços da clínica. Além de contribuírem para que a Clínica Alfa fosse referência em Saúde Dentária no País. Além de estreitar o relacionamento e ampliar sua carteira de clientes, possibilitou em um curto espaço de tempo aumentar o faturamento em cerca de $60 \%$, sobrevivendo à crise que o país está passando, onde empresas do mesmo setor estão fechando.

'Em tempos difíceis, ter um bom relacionamento com o cliente é a arma que uma organização precisa para se manter aberta', declarou o Presidente da Alfa. Foi possível também com o estudo criar um perfil dos clientes da clínica que são: homens e mulheres, entre 20 anos e 40 anos da classe B, C, que se preocupa com 
a saúde dentária e sua aparência. É possível observar de forma sucinta os benefícios da adoção do CRM e da Web 2.0 identificados no estudo: aumento do faturamento; conhecer o perfil do cliente; aumentar a carteira de clientes; fortalecer o relacionamento com o cliente; facilitar o trabalho dos profissionais; ter acesso às informações em qualquer lugar.

Os resultados só confirmam o que vêm sendo estudado por outros autores, a exemplo de Gallo et al. (2006) que diz que entre os benefícios da implantação do CRM no setor da saúde estão: a possibilidade do conhecimento do perfil do cliente e a determinação do perfil dos serviços de maior oferta pela Instituição. Com o resultado do estudo é possível concluir também que o diferencial competitivo no setor da saúde não está só na qualidade do serviço, mas também na gestão do negócio.

\section{REFERÊNCIAS}

ALKHATEEB, F. M.; CLAUSON, K. A.; KHANFAR, N. M.; LATIF, D. A.. Legal and regulatory risk associated with Web 2.0 adoption by pharmaceutical companies. Journal of Medical Marketing: Device, Diagnostic and Pharmaceutical Marketing, v.8, n.4, p.311-318, 2008.

AURILI, A.; PIERRE L.. O filósofo da cibercultura. São Paulo: Instituto Claro, 2013.

BRITO, A. E. M.; BRASILEIRO, F. V.; LEITE, C. E.; BURITI, A. C.. Comunicação Ethernet em Tempo-Real para uma Rede de Microcontroladores. In: CONGRESSO BRASILEIRO DE AUTOMÁTICA, 15. Anais. João Pessoa: UFPB, 2004

BULL, C.. Strategic issues in customer relationship management (CRM) implementation. Business Process Management Journal, v.9, n.5, p.592-602, 2003.

BUTTLE, F.. Customer relationship management: concepts and technologies. Logan: Routledge, 2009.

COOKE, M.; BUCKLEY, N.. Web 2.0: social networks and the future of market research. International Journal of Market Research, v.50, n.2, p.267, 2008.

GALLO, R.; MONKEN, S. F.. Sistemas de informação de marketing no setor saúde: aplicabilidade do gerenciamento do relacionamento do cliente. UNIrevista, v.3, n.1, 1-8, 2006.

GLICK, S.. CRM-More than just a software program. CPA Pratice Management Forum, v.3, p.11, 2007.

GURAU, C.; RANCHHOD, A.; HACKNEY, R.. Customer-centric strategic planning: Integrating CRM in online business systems. Information technology and management, v.4, n. 3, p.199-214, 2003.

KRAUS, M.; DALFOVO, O.. Sistemas de informação na saúde: uma aplicação do 'customer relationship management' (CRM) em uma pulmoclínica. Revista Eletrônica de Sistemas de Informação, v.5, n.3, 2006.
LAUDON, K. C.; LAUDON, J. P.. Sistema de informação com internet. 4 ed. Rio de Janeiro: Livros Técnicos e Científicos, 1999.

LEITE, C. R.; ARAUJO, B. G.; VALENTIM, R. A. D. M.; BRANDÃO, G. B.; GUEIRREIRO, A. M.. Novas tecnologias para automação hospitalar. Parnaíba: ERCEMAPI, 2009.

LIBERATO, M. M.. Fatores organizacionais que influenciam na adoção do CRM em empresas de pequeno e médio porte: um estudo de caso em empresas de Sergipe. In: SIMPÓSIO DE ENGENHARIA DE PRODUÇÃO, 22. Anais. Bauru, 2014.

MARTINEZ, D.; LEÃO, M. L.; STUMPF, M. K.. Informática médica. Brasília: CNPq, 1982.

MORAN, E.; GOSSIEAUX, F.. Marketing in a hyper-social world. Journal of Advertising Research, v.50, n.3, p.232-239, 2010.

NEVES, J. L.. Pesquisa qualitativa: características, usos e possibilidades. Caderno de pesquisas em administração, São Paulo, v.1, n.3, p.2, 1996.

PAYNE, A.. Handbook of CRM: achieving excellence in customer management. Logan: Routledge, 2006.

PEPPARD, J.. Customer relationship management (CRM) in financial services. European Management Journal, v.18, n.3, p.312-327, 2000.

SHIN, J. W.; CHA, D. Y.; LEE, K. J.; YOON, Y. R.. The web-based fuzzy patient monitor system. In: PROCEEDINGS OF THE ANNUAL INTERNATIONAL CONFERENCE OF THE IEEE, 22. Anais. Cancun: IEEE, 2000.

TEHRANI, N.. Everything you always wanted to know about CRM, but were afraid to ask. Call Center CRM Solutions, v.19, p.2-6, 2000.

URBANOWICZ, M.. Concept of Customer Relationship Management as an example of innovation in banking sector. Lisboa: Universidade Nova de Lisboa, 2008. 